\title{
GALACTIC AND EXTRAGALACTIC MAGNETIC FIELDS
}

\author{
RAINER BECK \\ Max-Planck-Institut für Radioastronomie, Auf dem Hügel 69, D-53121 Bonn, Germany
}

Received: 14 July 2000; Accepted: 15 November 2000

\begin{abstract}
The current state of research of the Galactic magnetic field is reviewed critically. The average strength of the total field derived from radio synchrotron data, under the energy equipartition assumption, is $6 \pm 2 \mu \mathrm{G}$ locally and about $10 \pm 3 \mu \mathrm{G}$ at $3 \mathrm{kpc}$ Galactic radius. These values agree well with the estimates using the locally measured cosmicray energy spectrum and the radial variation of protons derived from $\gamma$-rays. Optical and synchrotron polarization data yield a strength of the local regular field of $4 \pm 1 \mu \mathrm{G}$, but this value is an upper limit if the field strength fluctuates within the beam or if anisotropic fields are present. Pulsar rotation measures, on the other hand, give only $1.4 \pm 0.2 \mu \mathrm{G}$, a lower limit if fluctuations in regular field strength and thermal electron density are anticorrelated along the pathlength.

The local regular field may be part of a "magnetic arm" between the optical arms. However, the global structure of the regular Galactic field is not yet known. Several largescale field reversals in the Galaxy were detected from rotation measure data, but a similar phenomenon was not observed in external galaxies. The Galactic field may be young in terms of dynamo action so that reversals from the chaotic seed field are preserved, or a mixture of dynamo modes causes the reversals, or the reversals are signatures of largescale anisotropic field loops. The Galaxy is surrounded by a thick disk of radio continuum emission of similar extent as in edge-on spiral galaxies. While the local field in the thin disk is of even symmetry with respect to the plane (quadrupole), the global thick-disk field may be of dipole type. The Galactic center region hosts highly regular fields of up to milligauss strength which are oriented perpendicular to the plane.

A major extension of the data base of pulsar rotation measures and Zeeman splitting measurements is required to determine the structure of the Galactic field. Further polarization surveys of the Galactic plane at wavelengths of $6 \mathrm{~cm}$ or shorter may directly reveal the fine structure of the local magnetic field.
\end{abstract}

\section{Observational Methods}

Interstellar magnetic fields can be observed indirectly at optical, infrared, submillimeter and radio wavelengths. Extensive reviews of observational methods were given by Heiles (1976), Spoelstra (1977), Verschuur (1979) and Tinbergen (1996). Zeeman spectral-line splitting data are available for gas clouds, e.g. near the Galactic center (Section 6). Optical polarization data yield the large-scale structure of the magnetic field in the local spiral arm (Section 3.1), but small-scale features may be contaminated by scattered light which is unrelated to magnetic fields. Polarization observations in the infrared and submillimeter ranges are rapidly evolving (see e.g. Greaves

Space Science Reviews ...: 1-19, 2019.

(C) 2019 Kluwer Academic Publishers. Printed in the Netherlands. 
et al., 2000) and are expected to contribute to our knowledge about magnetic fields in the near future. Linearly polarized radio continuum emission at centimeter wavelengths and its Faraday rotation provide the most extensive and reliable information on large-scale interstellar magnetic fields in external galaxies (Beck et al., 1996; Beck, 2000) and in the Galactic center (Section 6). Information about the large-scale structure of the Galactic magnetic field near the Sun mainly comes from Faraday rotation measures of pulsars and extragalactic sources (Section 3) while polarization surveys of the Galactic plane at centimeter wavelengths reveal a wealth of small-scale magnetic structures.

Single-dish telescopes, like the 100-m Effelsberg dish, are powerful instruments in detecting weak, extended radio emission. To achieve sufficiently high resolution in external galaxies, interferometric (synthesis) telescopes are used (like the Westerbork Synthesis Radio Telescope, the Very Large Array, the Australia Telescope Compact Array) which, however, miss largescale structures so that the combination of interferometric with single-dish data is often required.

Interstellar magnetic fields are illuminated by cosmic-ray electrons, spiralling around the field lines and emitting synchrotron radiation, the dominant contribution to the diffuse radio continuum emission at centimeter and decimeter wavelengths. Synchrotron emission is intrinsically highly linearly polarized, $70-75 \%$ in a completely regular magnetic field. The observable degree of polarization in galaxies is reduced by Faraday (wavelengthdependent) depolarization in magnetized plasma clouds, by geometrical (wavelength-independent) depolarization due to variations of the magnetic field orientation across the telescope beam and along the line of sight, and by a contribution of unpolarized thermal emission which is dominating only in star-forming regions of the Galaxy. A map of the total radio intensity reveals the strength of the total interstellar magnetic field in the plane of the sky (averaged over the volume traced by the telescope beam), polarized intensity and polarization angle (see Figure 4) the strength and structure of the resolved regular field in the plane of the sky.

Polarization angles are ambiguous by $\pm 180^{\circ}$ and hence insensitive to field reversals. Imagine that a magnetic field without any regular structure (an isotropic random field) is compressed or stretched in one dimension. Emission from the resulting anisotropic field is linearly polarized with ordered vectors, but the field is incoherent, i.e. it reverses its direction frequently within the telescope beam. Here the polarization vectors just indicate anisotropy of the magnetic field distribution in the emission region.

The orientation of polarization vectors is changed in a magneto-ionic medium by Faraday rotation which is proportional to the product of the average density of thermal electrons and the strength of the regular field component along the line of sight. At centimeter wavelengths the Faraday ro- 
tation angle $(\Delta \chi)$ of the polarization vectors varies with $\lambda^{2} .\left(\Delta \chi=R M \lambda^{2}\right.$, where $R M$ is called the rotation measure, see Section 2.2.) Typical interstellar rotation measures of $\simeq 50 \mathrm{rad} / \mathrm{m}^{2}$ lead to $126^{\circ}$ rotation at $\lambda 21 \mathrm{~cm}$, $10^{\circ}$ at $\lambda 6 \mathrm{~cm}$ and $3^{\circ}$ at $\lambda 3 \mathrm{~cm}$. Below about $\lambda 3 \mathrm{~cm}$ Faraday rotation is generally small so that the $\mathbf{B}$-vectors (i.e. the observed $\mathbf{E}$-vectors rotated by $90^{\circ}$ ) directly trace the orientation of the regular field in the sky plane. However, Faraday rotation near the Galactic center is significant even at $\lambda 3 \mathrm{~cm}$ (Section 6). Maps of Faraday rotation measures (see Figures 3 and 5 ) give the strength and the direction of the average field components along the line of sight. $R M s$ are essential to distinguish between coherent and incoherent fields: In an incoherent (anisotropic) field the $R M s$ are random and show no large-scale structure (see Section 3.2).

At decimeter wavelengths Faraday depolarization significantly affects the polarized radio emission (Sokoloff et al., 1998). Differential Faraday rotation occurs within the synchrotron-emitting medium along the line of sight even if the magnetic field is completely regular. It leads to zero polarized intensity ("Faraday shadows") at certain wavelengths where the observed rotation of the polarization angle reaches multiples of $90^{\circ}$. Many "filaments" of vanishing polarized intensity, accompanied by $90^{\circ}$ jumps in polarization angle across the filament, were detected in the $\lambda 21 \mathrm{~cm}$ polarization survey out of the Galactic plane (Uyanıker et al., 1999), in high-resolution maps of the local Galactic emission at $\lambda 90 \mathrm{~cm}$ (Haverkorn et al., 2000) and in polarization maps of external galaxies (Beck, 2000). These do not indicate regions with zero strength of the regular field, but large changes in Faraday rotation measures across the "filament". Turbulent magnetic fields cause a dispersion in Faraday rotation measures which further depolarizes the emission. Even polarization maps of the Galactic plane at $\lambda 11 \mathrm{~cm}$ (Duncan etal., 1999) are affected by Faraday depolarization. The fine structure of the Galactic magnetic field may appear at wavelengths of $\leq \lambda 6 \mathrm{~cm}$, but such observations are difficult due to the low synchrotron intensities at short wavelengths.

\section{Magnetic Field Strength in the Galactic Disk}

\subsection{EQUIPARTITION}

The average strength of the total $\left\langle B_{\mathrm{t}, \perp}\right\rangle$ and the resolved regular field $\left\langle B_{\text {reg, }, \perp}\right\rangle$ in the plane of the sky can be derived from the total and polarized radio synchrotron intensity, respectively, if energy-density equipartition between cosmic rays and magnetic fields or minimum total energy density is assumed. Furthermore, the ratio $K$ between cosmic-ray protons and electrons (and its variation with particle energy), the synchrotron spectral index

$\alpha$ (i.e. flux density $S \propto \nu^{-\alpha}$ ), the extent of the radio-emitting region along 
the line of sight, and the volume filling factor of the field have to be known. Fortunately, the derived equipartition field strength depends on the power $1 /(\alpha+3)$ of each of these parameters so that even large uncertainties lead to only moderate errors in field strength.

The field strength may vary within the observed volume, e.g. due to compression by density waves, supernova remnants or flux freezing in gas clouds. In such cases the equipartition field strength is overestimated because $\left\langle B_{\mathrm{t}, \perp}^{3+\alpha}\right\rangle^{1 /(3+\alpha)}$ is larger than the true mean field $\left\langle B_{\mathrm{t}, \perp}\right\rangle$. For strong fluctuations $(\delta B /<B>\simeq 1)$ the equipartition strength is $\simeq 40 \%$ too large. If, on the other hand, the field is concentrated in filaments with a volume filling factor $f$, the equipartition/minimum-energy estimate is smaller than the true field strength in the filaments by a factor $f^{1 /(3+\alpha)}$.

The textbook equipartition/minimum-energy formulae use a fixed integration interval in radio frequency to determine the total energy density of cosmic-ray electrons. This procedure makes it difficult to compare field strengths between galaxies because a fixed frequency interval corresponds to different electron energy intervals if the field strengths are not the same. When instead a fixed integration interval in energy is used (say, from $300 \mathrm{MeV}$ to infinity), the minimum-energy and energy equipartition estimates give very similar values for $\left\langle B_{\mathrm{t}, \perp}^{3+\alpha}\right\rangle$, where $\alpha$ is typically $\simeq 0.9$.

The mean equipartition strength of the total field (using $K=100$ ) for a sample of 74 spiral galaxies (corrected for the inclination of each galaxy) is $\left\langle B_{\mathrm{t}}\right\rangle=9 \mu \mathrm{G}$ with a standard deviation of $3 \mu \mathrm{G}$ (Niklas, 1995). The values for the Galaxy (Figure 1) fit well into this range. In nearby galaxies the average equipartition strength of the total field in the galactic disks ranges between $\left\langle B_{\mathrm{t}}\right\rangle \simeq 6 \mu \mathrm{G}$ in radio-faint spiral galaxies like M31 and M33, and $\simeq 15 \mu \mathrm{G}$ in grand-design galaxies like M51, M83 and NGC 6946. In spiral arms the total field strength is $10-20 \mu \mathrm{G}$ over kpc scales, but locally can reach $\simeq 1 \mathrm{mG}$ in molecular clouds compressed by supernova shocks (Brogan et al., 2000) and in filaments near the Galactic center (see Section 6).

Figure 1 shows the radial variation of the equipartition field strength in the Galaxy, obtained from the radial variation of the total synchrotron emission at $408 \mathrm{MHz}(\lambda 74 \mathrm{~cm})$ derived by Beuermann et al. (1985) from the survey of Haslam etal. (1974). The total field strength is $6 \pm 2 \mu \mathrm{G}$ locally and $10 \pm 3 \mu \mathrm{G}$ at $3 \mathrm{kpc}$ Galactic radius.

The equipartition/minimum-energy assumption is under continuous debate.

Firstly, this assumption may be valid only on long time/space scales where equilibrium conditions can develop, but may break down on short/small scales. Secondly, synchrotron or inverse-Compton energy losses of the cosmicray electrons cause a steepening of the $\mathrm{GeV}$ electron spectrum so that $K$ increases with increasing energy. This leads to an overestimate of the field strength (Pohl, 1993). However, there is little indication for a steepening of 


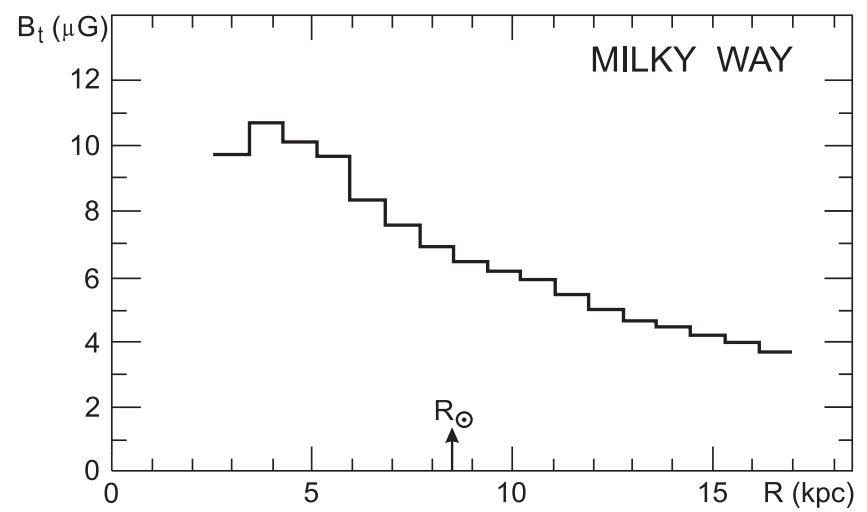

Figure 1. Strength of the total magnetic field in the Galaxy, averaged from the deconvolved surface brightness of the synchrotron emission at $408 \mathrm{MHz}$ (Beuermann et al., 1985), assuming energy equipartition between magnetic field and cosmic ray energy densities (Berkhuijsen, personal communication). The accuracy is about $30 \%$. The Sun is assumed to be located at $\mathrm{R}=8.5 \mathrm{kpc}$.

the radio synchrotron spectrum at frequencies beyond $1 \mathrm{GHz}$ (and hence the electron spectrum beyond $\simeq 3 \mathrm{GeV}$ ) of spiral galaxies (Niklas and Beck, 1997).

In the Galaxy the accuracy of the equipartition assumption can be tested because we have independent information about the local cosmic-ray electron energy density from direct measurements and about the cosmic-ray proton distribution from $\gamma$-ray data. Combination of the radio synchrotron emission, the local cosmic-ray electron density and diffuse continuum $\gamma$-rays yields a local strength of the total field of $6 \pm 1 \mu \mathrm{G}$ (Strong et al., 2000), almost the same value as derived from energy equipartition (Figure 1). Even the radial variation of the equipartition field strength in Figure 1 is in excellent agreement with that derived by Strong et al. (2000).

Synchrotron polarization observations in the Galaxy at decimeter wavelengths imply a ratio of regular to total field strengths within about a $\mathrm{kpc}$ from the Sun of $<B_{\mathrm{reg}} / B_{\mathrm{t}}>\simeq 0.6$ (Berkhuijsen, 1971; Brouw and Spoelstra, 1976; Heiles, 1996). The radio emission along the local spiral arm requires that the strength of the regular field components is similar or slightly less than that of the turbulent field, or $\left\langle B_{\mathrm{reg}} / B_{\mathrm{t}}\right\rangle \leq 0.7$ (Phillipps et al., 1981). For $\left\langle B_{\mathrm{t}}\right\rangle=6 \pm 2 \mu \mathrm{G}$ these results give $4 \pm 1 \mu \mathrm{G}$ for the large-scale regular component.

Experience from external galaxies shows that the regular field is weaker and the turbulent field is stronger in the spiral arms, probably due to field tangling by star-forming processes and the expansion of supernova remnants. In interarm regions the regular field can be much stronger than the turbulent field. 
The strength of the large-scale regular fields $B_{\text {reg }}$ in spiral galaxies (observed with a spatial resolution of a few $100 \mathrm{pc}$ ) is typically $1-5 \mu \mathrm{G}$, up to $\simeq 13 \mu \mathrm{G}$ in an interarm region of NGC 6946 hosting an exceptionally strong regular field (Beck and Hoernes, 1996) (see Figure 4).

Note that polarized emission does not distinguish between coherent and inhoherent (anisotropic) fields (Section 1) so that the strength of the coherent regular field may be lower than the equipartition estimates.

\subsection{Pulsars}

The Faraday rotation measure is defined as $R M=k \int n_{\mathrm{e}} B_{\|} d l$, where $B_{\|}$ is the component of the regular field $B_{\text {reg }}$ along the line of sight. Measurement of pulsar $R M s$ together with their dispersion measures $D M=\int n_{\mathrm{e}} d l$ allows an estimate of $B_{\|}$of the local Galactic field (the "standard estimate"): $<B_{\|}>=R M D M^{-1} k^{-1}$.

The strength of the local regular field is $\left\langle B_{\text {reg }}\right\rangle=1.4 \pm 0.2 \mu G$ (Rand and Lyne, 1994; Han and Qiao, 1994; Indrani and Deshpande, 1998), less than the equipartition estimate (Section 2.1).

The above field estimate suffers from various problems:

- The "standard estimate" assumes that the variations of $B_{\|}$and $n_{\mathrm{e}}$ are uncorrelated along the line of sight. If however these quantities are correlated, we get $R M / D M=k<B_{\|}>\left(1+\delta B_{\|}^{2} /<B_{\|}>^{2}\right)$.

If $\delta B_{\|} /<B_{\|}>\simeq 0.5: \quad<B_{\|}>=0.8 R M D M^{-1} k^{-1}$.

Here the standard estimate is too large and even increases the discrepancy to the equipartition value.

- Observations of external galaxies indicate that $B_{\mathrm{reg}}$ and $n_{\mathrm{e}}$ are anticorrelated on kpc scales (Beck and Hoernes, 1996). $B_{\text {reg }}$ is strongest in the interarm regions and weaker in the spiral arms where $n_{\mathrm{e}}$ is largest (see Figure 4). Even if $\left\langle n_{\mathrm{e}}\right\rangle$ and $\left\langle B_{\text {reg }}\right\rangle$ are uncorrelated in different regions of the sky, the fluctuations $\delta n_{\mathrm{e}}$ and $\delta B$ can still be anticorrelated on small scales along the line of sight (pressure balance), e.g. $\delta B_{\|} /<B_{\|}>=-\delta n_{\mathrm{e}} /<n_{\mathrm{e}}>$. For small fluctuations we get:

$R M / D M=k<B_{\|}>\left(1-\delta B_{\|}^{2} /<B_{\|}>^{2}\right)$. If $\delta B_{\|} /<B_{\|}>\simeq 0.5: \quad<B_{\|}>=1.3 R M D M^{-1} k^{-1}$.

Here the standard estimate is too small. The larger the anticorrelated fluctuations, the smaller the observed rotation measure.

- If there are $N$ field reversals along the line of sight, the standard estimate is $\simeq(N+1)$ times too small. The pulsar $R M$ data have been corrected for detected reversals, but there may be more hidden reversals.

Hence $<B_{\text {reg }}>$ derived from pulsar $R M s$ may be an underestimate. On the other hand, equipartition field strengths based on polarized synchrotron emission may be too large (Section 2.1). Anisotropic fields (produced e.g. by shear motions, elongated supernova remnants, or Parker loops) may explain 
the discrepancy in field strengths and also some of the reversals near the Sun (see Section 3.2).

\section{Structure of the Regular Field in the Galactic Disk}

Highly polarized radio emission from the local spiral arm was observed at decimeter wavelengths with the Dwingeloo single-dish telescope (e.g. Berkhuijsen, 1971; Brouw and Spoelstra, 1976). A substantial fraction of the Galactic magnetic field must be regular on various spatial scales. With higher spatial resolution more fine structure of the field can be resolved. Surveys of the Galactic plane with the Parkes telescope (Duncan et al., 1997) and the Effelsberg telescope (Junkes et al., 1987; Duncan et al., 1999; Uyanıker et al., 1999) revealed high degrees of polarization in small nearby regions where the regular field dominates. In external galaxies average fractional polarizations, observed with several $100 \mathrm{pc}$ spatial resolution, are less than a few percent in central regions and spiral arms, but $20-40 \%$ in between the spiral arms and in outer regions where the field is regular on very large scales (Beck et al., 1996; Beck, 2000).

\subsection{Pitch Angles}

The Sun is located between two spiral arms, the Sagittarius/Carina and the Perseus arms, as delineated e.g. by thermal electrons (Taylor and Cordes, 1993; Vallée, 1996). The local (or Orion) arm seen in optical and HI emission is probably an interarm spur. The mean pitch angle of the local and neighboring spiral arms is $\simeq-18^{\circ}$ for the stars and $\simeq-13^{\circ}$ for the gas (see compilation by Vallée, 1995). However, starlight polarization and pulsar $R M$ data (Figure 2) give a significantly smaller pitch angle $\left(\simeq-8^{\circ}\right)$ for the local magnetic field (Heiles, 1996; Han and Qiao, 1994; Indrani and Deshpande, 1998; Han et al., 1999a).

The pitch angle of the "magnetic arms" in the spiral galaxy NGC 6946 (see Figure 4) is $10-20^{\circ}$, smaller than that of the optical arms (Rohde et al., 1999). The local Galactic field may also form a "magnetic arm" with a small pitch angle, located between two optical arms (Han and Qiao, 1996). The amplitude of the $R M$ variation with azimuthal angle in NGC 6946 (see Figure 5 ) is $\simeq 70 \mathrm{rad} / \mathrm{m}^{2}$ at an inclination of $30^{\circ}$. Looking along the field, we

would observe $R M \simeq 140 \mathrm{rad} / \mathrm{m}^{2}$, similar to $R M s$ of extragalactic sources seen along the local arm (Figure 3 ). 


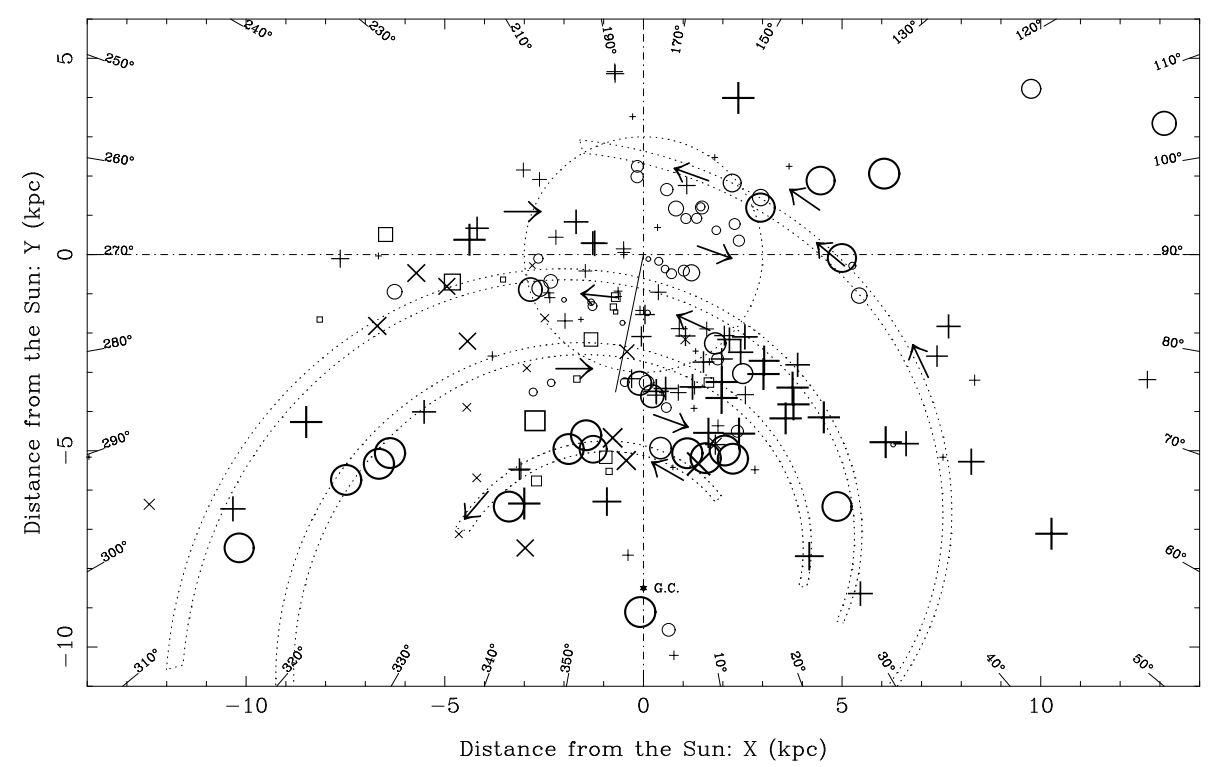

Figure 2. The distribution of the $R M s$ of pulsars within $8^{\circ}$ of the Galactic plane. Positive $R M s$ are shown as crosses, negative $R M s$ as circles. The most recent $R M$ data are indicated by $\mathrm{X}$ and open squares. The symbol sizes are proportional to the square root of $|R M|$, with the limits of 5 and $250 \mathrm{rad} / \mathrm{m}^{2}$. The directions of the bisymmetric field model are given as arrows. The approximate location of four spiral arms is indicated as dotted lines. The dotted circle has a radius of $3 \mathrm{kpc}$ (from Han et al., 1999a).

\subsection{Field Reversals}

The most interesting property of the Galactic magnetic field is the existence of reversals. A reversal inside the solar radius, between the local and the Sagittarius arm, was already detected from rotation measures of polarized extragalactic radio sources (Simard-Normandin and Kronberg, 1980). A recent analysis with the wavelet technique (Frick et al., 2000) confirmed this reversal at $\simeq 0.6 \mathrm{kpc}$ from the Sun and indicates another reversal in the outer galaxy. Both reversals are also seen in the data of pulsar RMs (Rand and Lyne, 1994; Han and Qiao, 1994). Two more reversals, one further inside and one further outside, were suggested by Han et al. (1999a).

Several explanations of the field reversals were proposed. (a) Dynamos prefer to generate axisymmetric modes without reversals, but their growth time can be quite long so that elongated field loops with reversals may remain from the chaotic seed field (Poezd et al., 1993). (b) The dynamo may generate higher modes, e.g. a bisymmetric one with large-scale reversals from one magnetic arm to the next. To account for several reversals along Galactic radius, a bisymmetric magnetic spiral with a small pitch angle $\left(\simeq-7^{\circ}\right)$ is needed (Han and Qiao, 1994; Indrani and Deshpande, 1998). 


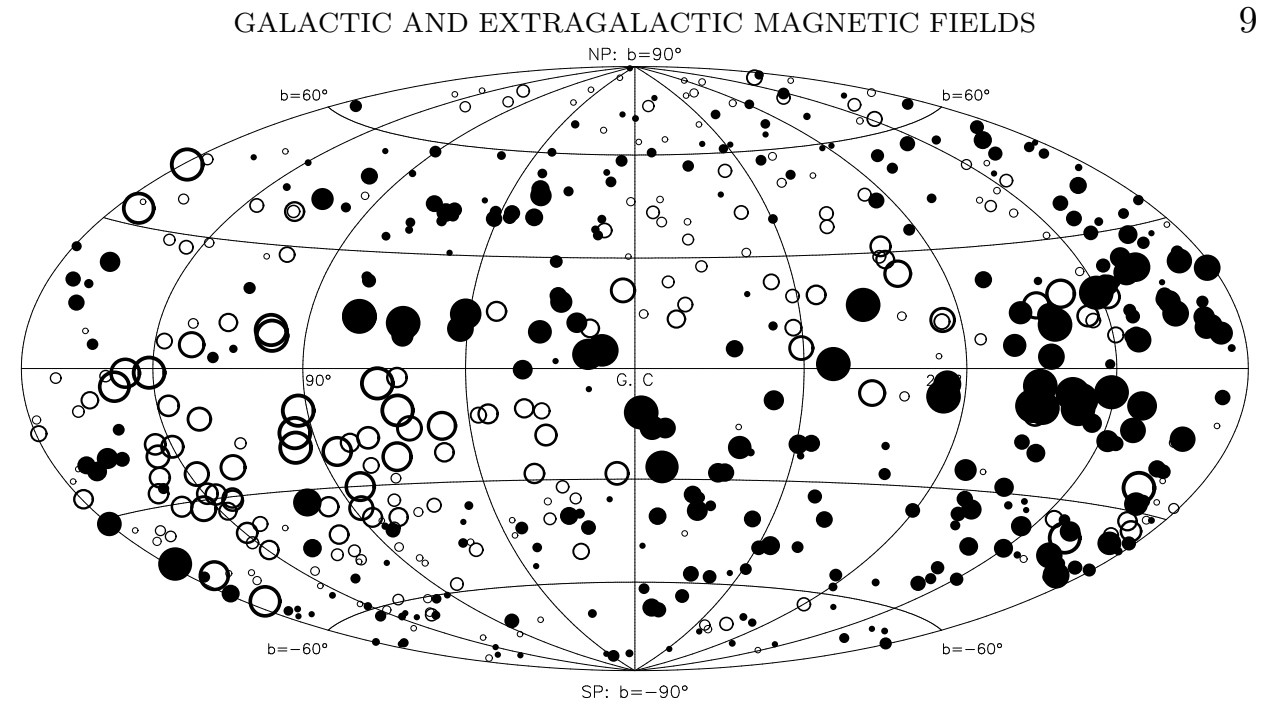

Figure 3. The distribution of the RMs of extragalactic radio sources. Filled circles indicate positive $R M s$, open circles negative $R M s$. The area of the circles is proportional to $|R M|$ within limits of 5 and $150 \mathrm{rad} / \mathrm{m}^{2}$ (from Han et al., 1997).

Figure 2 shows the distribution of $R M s$ of low-latitude pulsars, projected onto the Galactic plane, and the bisymmetric model by Han et al. (1999a). Some data disagree with the model. This may be due to local disturbances of the field e.g. by supernova remnants (Vallée, 1996), or the model is too simple. More pulsar $R M$ data are needed to obtain a better sampling of the field structure. (c) Nonlinear dynamo models revealed a mixture of magnetic modes, while the dominance of the bisymmetric mode is very difficult to obtain. A model based on the rotation curve of M51 and a spiral modulation generated a large-scale reversal near the corotation radius in one half of the galaxy where the bisymmetric field can be trapped by the spiral pattern over the galaxy's lifetime (Bykov et al., 1997, see below). However, no reversals at other radii appeared. (d) Large-scale anisotropic field loops may be produced by stretching or compressing (see below).

In external galaxies, data sampling is much denser than in the Galaxy. High-resolution maps of Faraday rotation, which measure the RMs of the diffuse polarized synchrotron emission, are available for a couple of spiral galaxies (Beck et al., 1996; Beck, 2000). It is striking that only very few field reversals have been detected in spiral galaxies where the spatial resolution is better than $1 \mathrm{kpc}$. The observed disk field of M51 can be described by a mixture of axisymmetric and bisymmetric components which may mimic a reversal for an observer located within the disk (see Figure 8a in Berkhuijsen et al., 1997, compare with Figure 6 in Bykov et al., 1997). In NGC 2997 a reversal between the disk field and the central region occurs at about $2 \mathrm{kpc}$ 
radius (Han et al., 1999b), but no reversal like that seen in the Milky Way was found in the outer disk of any galaxy. Some evidence for a dominating bisymmetric field structure was found from the large-scale azimuthal pattern variation of $R M s$ in M33 and M81 (Krause, 1990), but with recently improved observations of these galaxies the evidence for M33 has not been confirmed (Fletcher et al., 2000).

The discrepancy between Galactic and extragalactic data may be due to the different volumes traced by the observations. Results in the Galaxy are based on pulsar $R M s$ which trace only the warm ionized medium near the plane, while extragalactic results are based on $R M s$ of the diffuse polarization emission integrated over the whole volume occupied by regular fields and thermal gas. Reversals in a thin disk would be difficult to detect in external galaxies - but why should reversals occur only near the plane?

With the limited number of pulsar $R M s$ it is not sure whether the reversals in the Galaxy really continue over a substantial part of the disk. Instead, they may be relics of the protogalactic seed field or part of a largescale anisotropic field, as indicated from observations in external galaxies. Figure 4 shows the observed distribution of polarized synchrotron emission in the galaxy NGC 6946 hosting a grand-design spiral field. The large-scale regular fields are restricted to the interarm region between the optical arms while the total field in the optical arms is strong, but tangled. The map of $R M s$ (Figure 5) can only give data where the signal-to-noise ratio of polarized intensities are large enough. A large-scale pattern is apparent: $R M s$ are predominantly positive in the northern half and predominantly negative in the southern half so that the field points inwards (towards the galactic center) everywhere. There is no large-scale reversal between the outer and the inner "magnetic arms" which are about $3 \mathrm{kpc}$ apart. On smaller scales, $R M s$ fluctuate significantly with a mean $\mathrm{rms}$ of $30-50 \mathrm{rad} / \mathrm{m}^{2}$, larger than expected from instrumental noise. Furthermore, the spatial scale of these fluctuations is about $1 \mathrm{kpc}$, large than that of the turbulent field and that of instrumental noise which should have a spatial scale of the telescope resolution which corresponds to $\simeq 400 \mathrm{pc}$ in NGC 6946. $R M$ variations in other spiral galaxies are similar, probably signature of $\mathrm{kpc}-$-scale fluctuations in the field structure (anisotropic fields, see Section 2.2). For example, a magnetic loop was identified in M31 and interpreted as a Parker loop (Beck et al., 1989).

Figure 5 gives some idea about what an observer inside NGC 6946 may see. The large-scale $R M$ pattern is detectable only for sufficiently long pathlengths, e.g. along the local magnetic arm in the Galaxy or along the inner Sagittarius arm. On smaller scales fluctuations in $R M$ with frequent reversals may dominate. Indeed, regions with the same sign of $R M$ of several $\mathrm{kpc}$ in diameter are visible in Figure 2. We need a realistic model of the field which can be used to predict what the internal observer would see, given a 


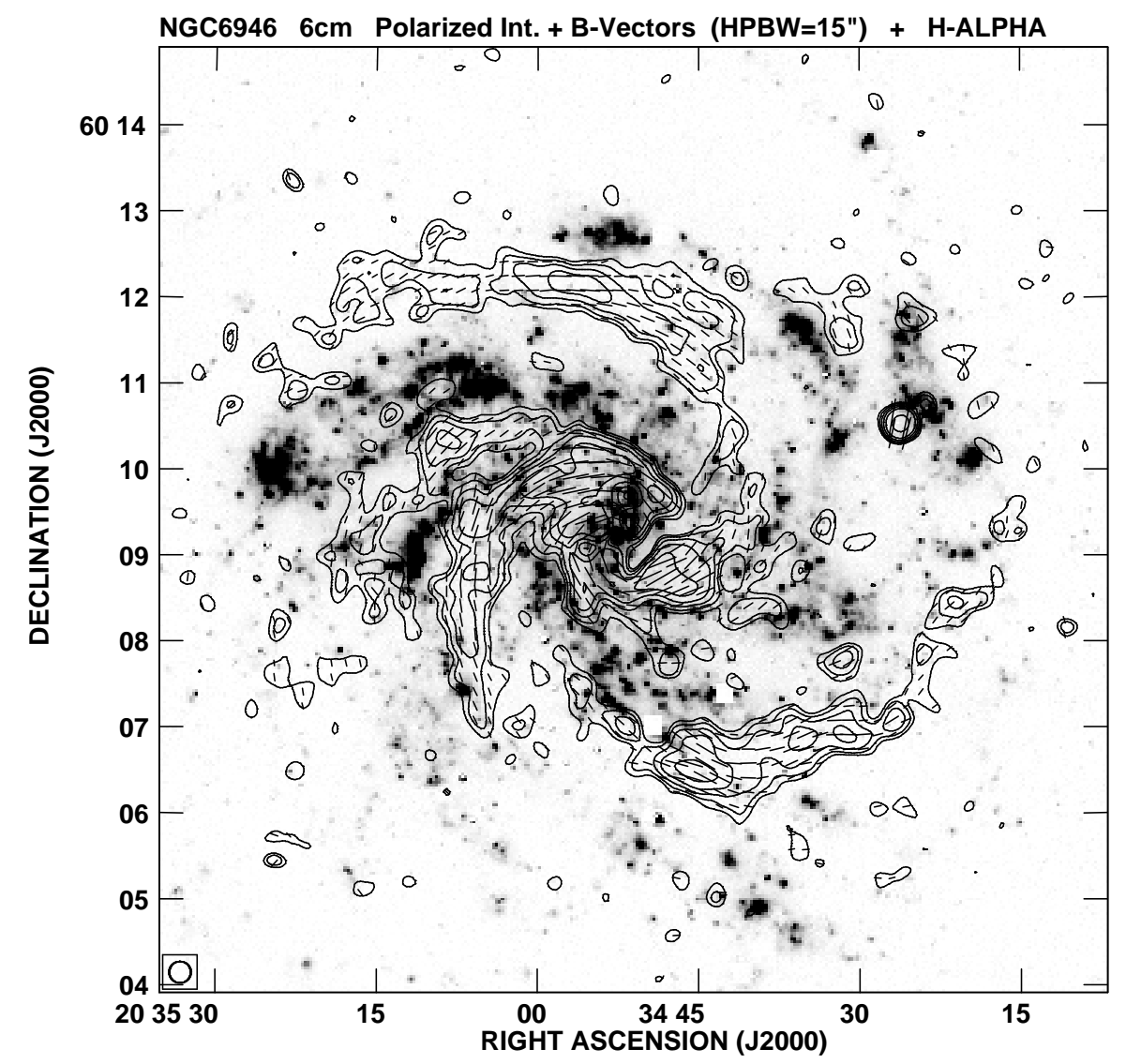

Figure 4. Polarized radio emission and B-vectors of the spiral galaxy NGC 6946 at $\lambda 6 \mathrm{~cm}$, combined from observations with the VLA and Effelsberg telescopes (from Beck et al. (1996) and overlaid onto an $\mathrm{H} \alpha$ image of Ferguson et al. (1998)). The angular resolution of the radio image is 15 arcsec.

limited number of pathlengths to pulsars. Sensitive Zeeman splitting data of a large number of clouds will also help.

\section{Turbulent Fields}

The Galactic magnetic field cannot be completely regular because we still observe synchrotron emission when looking at the field along the local arm so that the regular field component in the sky plane vanishes. Starlight and synchrotron polarization data give a ratio of regular to turbulent field strengths of $\simeq 0.6-1.0$ (Section 2.1). With $B_{\text {reg }} \simeq 4 \mu \mathrm{G}$ the strength of the 


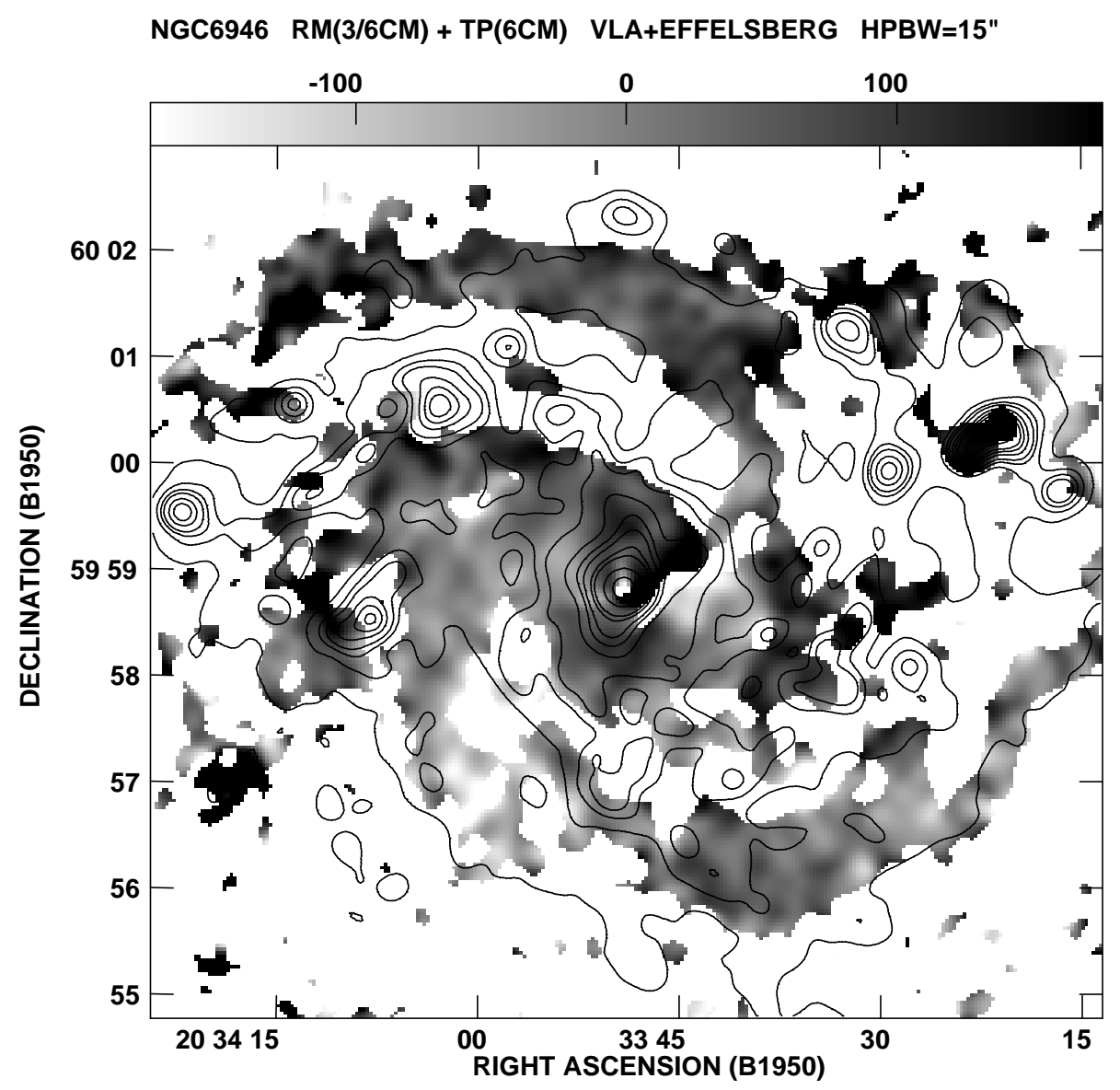

Figure 5. Grey-scale map of the Faraday rotation measures of NGC 6946 between $\lambda 3 \mathrm{~cm}$ and $\lambda 6 \mathrm{~cm}$, overlayed onto a total intensity map at $\lambda 6 \mathrm{~cm}$. The data were obtained from combined polarization observations with the VLA and Effelsberg telescopes (Beck, unpublished). The angular resolution is 15 arcsec.

local turbulent field is $B_{\text {turb }} \simeq 5 \mu \mathrm{G}$. This agrees well with the estimates from the dispersion of pulsar RMs (Rand and Kulkarni, 1989; Ohno and Shibata, 1993). Note that according to experience from external galaxies the turbulent field is larger than the regular field in the spiral arms.

Magnetic turbulence occurs over a large spectrum of scales (Minter and Spangler, 1996). The largest scale of the turbulent field was determined from pulsar $R M s$ as $l_{\text {turb }} \simeq 55 \mathrm{pc}$ (Rand and Kulkarni, 1989) or $l_{\text {turb }} \simeq 10-100 \mathrm{pc}$ (Ohno and Shibata, 1993). $l_{\text {turb }}$ can also be derived from the depolarization by turbulent fields at centimeter radio wavelengths; the result is $l_{\text {turb }} \simeq$ $25 \mathrm{pc} \times f^{1 / 3}$ for the galaxy NGC 6946 where $f$ is the filling factor of the turbulent cells (Beck et al., 1999). At decimeter radio wavelengths turbulent 
fields also cause Faraday dispersion. Applying standard formulae (Sokoloff et al., 1998) yields $l_{\text {turb }} \simeq 7 \mathrm{pc} / f$ (Beck et al., 1999). The two estimates agree for $l_{\text {turb }} \simeq 20 \mathrm{pc}$ and $f \simeq 0.4$.

The type of turbulence (3-D or 2-D, or else) can be derived from the structure function (Minter and Spangler, 1996). The application to rotation measures and emission measures $\left(E M=\int n_{\mathrm{e}}^{2} d l\right)$ for the same field near the Galactic plane indicated 2-D turbulence (thin sheets or filaments), an anisotropic turbulent field. Application to external galaxies gave similar results (Beck etal., 1999).

Future $\lambda 6 \mathrm{~cm}$ polarization surveys of the Galactic plane may show the filamentary structure of the Galactic field directly.

\section{Magnetic Fields in Thick Disks and Halos}

Synchrotron emission can be followed until high Galactic latitudes indicating the existence of a radio halo or a thick disk. Its vertical full equivalent thickness is $3.0 \pm 0.2 \mathrm{kpc}$ near the Sun (Beuermann et al., 1985, scaled to a distance to the Galactic center of $8.5 \mathrm{kpc}$ ) which corresponds to an exponential scale height of $h_{\mathrm{syn}}=1.5 \pm 0.1 \mathrm{kpc}$. This is also the lower limit for the scale height of the total magnetic field. In case of equipartition between cosmic rays and magnetic fields and a synchrotron spectral index of $\alpha \simeq 1$, the thickness of the distribution of the total field is $(3+\alpha) \simeq 4$ times larger than that of the synchrotron disk, i.e. $h_{B} \simeq 6 \mathrm{kpc}$. The scale height of the regular field in the warm ionized medium is $h\left(B_{\text {reg }}\right) \simeq 1.5 \mathrm{kpc}$, determined from pulsar RMs (Han and Qiao, 1994).

In external galaxies seen edge-on the scale height of the thick synchrotron disks is $\simeq 2 \mathrm{kpc}$ (Dumke and Krause, 1998), similar to that of the Galaxy. The degree of polarization increases with increasing distance from the plane, due to a decrease of the thermal contribution and of the depolarization by turbulent fields away from the plane. Hence the scale height of the regular field is difficult to determine.

The local Galactic field is oriented mainly parallel to the plane, with a vertical component of only $B_{z} \simeq 0.2-0.3 \mu \mathrm{G}$ (Han and Qiao, 1994). This agrees well with the results from external galaxies (Dumke et al., 1995).

Plane-parallel fields are a signature of symmetric dynamo modes where the toroidal component has the same sign above and below the plane and the poloidal field is of quadrupole type. $R M s$ of extragalactic sources (see Figure 3) as well as pulsar RMs have the same sign above and below the plane for Galactic longitudes between $90^{\circ}$ and $270^{\circ}$. Thus the local field is part of a large-scale symmetric (quadrupole) field structure in the thin disk. However, towards the inner Galaxy (between $270^{\circ}$ and $90^{\circ}$ longitude) the signs are opposite above and below the plane. This may be due to local spurs 
and field loops (Vallée, 1996), but may also indicate a global antisymmetric (dipole) mode in the thick disk (Andreasyan and Makarov, 1988) or in the inner galaxy (Han et al., 1997) where the field component perpendicular to the plane is strong, as observed near the Galactic center (Section 6).

Signs of an antisymmetric field were found in the huge radio halo of the galaxy NGC 4631 (Beck, 2000). The poloidal field component is of dipole type and allows fast propagation of cosmic rays into the halo. As a consequence, the radio halo of NGC 4631 is larger than that of all other edge-on galaxies observed so far.

The existence of quadrupole and/or dipole-type fields in the Galaxy is of vital interest for cosmic-ray physics. An extension of the $R M$ data base is required to clarify this question.

\section{Magnetic Fields Near the Galactic Center}

At 0.2 Galactic longitude (i.e. 30 pc from the Galactic center) a system of polarized filaments (the "Arc") extends to at least $3^{\circ}(450 \mathrm{pc})$ perpendicular to the plane (Seiradakis etal., 1985; Tsuboi etal., 1986; Pohl etal., 1992; Haynes et al., 1992). $\lambda 9 \mathrm{~mm}$ Effelsberg polarization observations (Figure 6) show that the regular magnetic field is almost perfectly aligned along the filaments. The standard equipartition estimate cannot be applied to derive the field strength because the electron spectrum appears monoenergetic. Using the fact that the filaments remain straight in the surrounding cloudy medium, the field strength was estimated to be about $1 \mathrm{mG}$ (Morris, 1994). Acceleration and lifetime arguments led to a similar estimate (Reich et al., 2000). This would allow confinement of high-energy cosmic rays, but requires an effective acceleration process, e.g. via reconnection (Lesch and Reich, 1992). A monoenergetic electron spectrum can also be provided by shock

acceleration if the particle escape time is much longer than the acceleration time (Schlickeiser, 1984).

Also in a few dense gas clouds in front of the Galactic center (about 2 pc north of the Galactic center) field strengths in the milligauss range were derived from Zeeman measurements (Plante et al., 1994; Yusef-Zadeh etal., 1996). On the other hand, low observed bandwidth depolarization restricts the average field in the SgrA complex to less than $0.4 \mathrm{mG}$ (Reich, 1994). The non-detection of the Zeeman effect in the $\mathrm{OH}$ lines (Uchida and Güsten, 1995) also indicates a relatively weak general magnetic field into which bundles or clouds with strong fields are embedded.

Vertical fields in the central regions were detected in several spiral galaxies (Hummel etal., 1983), but were mostly assigned to nuclear activity. Radio polarization observations of edge-on galaxies with high resolution may show similarities to the central region of the Galaxy. 

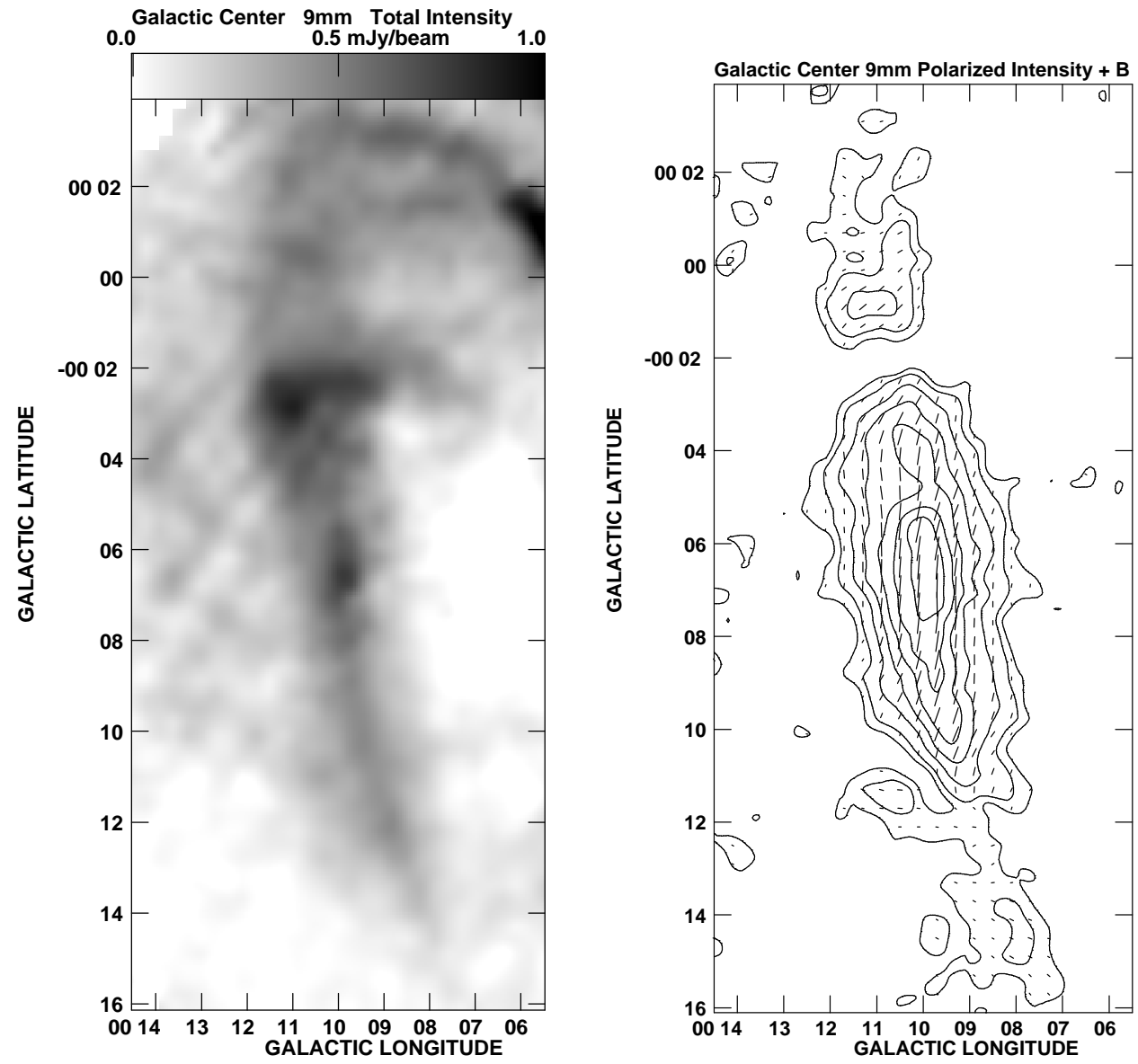

Figure 6. Total radio emission (left) and polarized emission with B-vectors (right) from the inner part of the "Arc" located 0.2 east from the Galactic center, observed at $\lambda 9 \mathrm{~mm}$ with the Effelsberg telescope (from Lesch and Reich, 1992). The angular resolution is 36 arcsec.

Recently a filament parallel to the Galactic plane, comprised of parallel strands, has been found 1.5 from the center (Lang et al., 1999), possibly linking the central with the global field (Section 5).

\section{Summary}

- The strength of the total magnetic field in the Galaxy is $6 \pm 2 \mu \mathrm{G}$ locally and $10 \pm 3 \mu \mathrm{G}$ at $3 \mathrm{kpc}$ Galactic radius. The local equipartition value agrees well with that derived using direct measurements of cosmic rays and $\gamma$-rays. 
- The strength of the local regular field is $4 \pm 1 \mu \mathrm{G}$, based on optical and synchrotron polarization data, while pulsar rotation measures give a more than twice lower value. The optical/synchrotron data may overestimate the regular field strength due to the presence of anisotropic fields, while the pulsar value may be an underestimate due to anticorrelated fluctuations in regular field strength and in thermal electron density.

- The local regular field may be part of a "magnetic arm" between the optical arms. The global structure of the Galactic field is unknown.

- No large-scale field reversals similar to those observed in the Galaxy were found in external galaxies. Some of the Galactic reversals may be due to large-scale anisotropic field loops. More pulsar $R M$ and Zeeman splitting data are needed.

- Polarization surveys of the Galactic plane at decimeter wavelengths show a wealth of details which are mostly due to variations of Faraday rotation measures in the foreground medium. In order to directly observe the fine structure of the field, surveys at wavelengths $\leq 6 \mathrm{~cm}$ are required.

- The Galaxy is surrounded by a thick radio disk with a scale height of $\simeq 1.5 \mathrm{kpc}$, similar to that of edge-on spiral galaxies.

- The local Galactic field in the thin disk is of even symmetry with respect to the plane (quadrupole), but a global dipole field in the thick disk is possible.

- The Galactic center region hosts highly regular fields of up to milligauss strength which are concentrated in narrow bundles, oriented perpendicular to the plane.

\section{Acknowledgements}

The author thanks Dr. E. M. Berkhuijsen for providing Figure 1 and for careful reading of the manuscript. Drs. JinLin Han, Wolfgang Reich, John Seiradakis, Steve Spangler, Andy Strong and Richard Wielebinski are acknowledged for many useful comments.

\section{References}

Andreasyan, R. R., and Makarov, A. N.: 1988, 'Model of Two-component Magnetic Field of the Galaxy', Astrophysics 28, 247-253.

Beck, R.: 2000, 'Magnetic Fields in Normal Galaxies', Phil. Trans. R. Soc. Lond. A 358, $777-796$.

Beck, R., and Hoernes, P.: 1996, 'Magnetic Spiral Arms in the Galaxy NGC 6946', Nature 379, 47-49. 
Beck, R., Loiseau, N., Hummel, E., Berkhuijsen, E. M., Gräve, R., Wielebinski, R.: 1989, 'High-resolution Polarization Observations of M31 I. Structure of the Magnetic Field in the Southwestern Arm', Astron. Astrophys. 222, 58-68.

Beck, R., Brandenburg, A., Moss, D., Shukurov, A., Sokoloff, D.: 1996, 'Galactic Magnetism: Recent Developments and Perspectives', Ann. Rev. Astron. Astrophys. 34, 155-206.

Beck, R., Berkhuijsen, E. M., Uyanıker, B.: 1999, 'Studies of Turbulent Interstellar Magnetic Fields Using Radio Emission', in M. Ostrowski and R. Schlickeiser (eds.), Plasma Turbulence and Energetic Particles in Astrophysics, Obserwatorium Astronomiczne, Kraków, 1999, pp. 5-17.

Berkhuijsen, E. M.: 1971, 'A Survey of the Continuum Radiation at $820 \mathrm{MHz}$ between Declinations $-7^{\circ}$ and $+85^{\circ}$, Astron. Astrophys. 14, 359-386.

Berkhuijsen, E. M., Horellou, C., Krause, M., Neininger, N., Poezd, A. D., Shukurov, A., Sokoloff, D. D.: 1997, 'Magnetic Fields in the Disk and Halo of M51', Astron. Astrophys. 318, 700-720.

Beuermann, K., Kanbach, G., Berkhuijsen, E. M.: 1985, 'Radio Structure of the Galaxy: Thick Disk and Thin Disk at $408 \mathrm{MHz}$ ', Astron. Astrophys. 153, 17-34.

Brogan, C.L., Frail, D.A., Goss, W.M., Troland, T.H.: 2000, 'OH Zeeman Magnetic Field Detections Toward Five Supernova Remnants Using the VLA', Astrophys. J. 537, 875-890.

Brouw, W. N., Spoelstra, T. A. Th.: 1976, 'Linear Polarization of the Galactic Background at Frequencies Between 408 and $1411 \mathrm{MHz}$. Reductions', Astron. Astrophys. Suppl. 26, 129-146.

Bykov, A., Popov, V., Skukurov, A., Sokoloff, D.: 1997, 'Anomalous Persistence of Bisymmetric Magnetic Structures in Spiral Galaxies', Mon. Not. R. Astron. Soc. 292, $1-10$.

Dumke, M., and Krause, M.: 1998, 'Radio and Polarization Properties in the Disk and Halo of Edge-on Spirals', in D. Breitschwerdt, M. J. Freyberg, and J. Trümper (eds.), The Local Bubble and Beyond, Springer-Verlag, 1998, pp. 555-558.

Dumke, M., Krause, M., Wielebinski, R., Klein, U.: 1995, 'Polarized Radio Emission at $2.8 \mathrm{~cm}$ from a Selected Sample of Edge-on Galaxies', Astron. Astrophys. 302, 691-703.

Duncan, A. R., Haynes, R. F., Jones, K. L., Stewart, R. T.: 1997, 'Polarized Radio Emission Over the Southern Galactic Plane at 2.4 GHz', Mon. Not. R. Astron. Soc. 291, 279295.

Duncan, A. R., Reich, P., Reich, W., Fürst, E.: 1999, 'Polarimetric Structure in the First Galactic Quadrant from the 2.695 GHz Effelsberg Survey', Astron. Astrophys. 350, 447-456.

Ferguson, A.M. N., Wyse, R.F.G., Gallagher, J.S., Hunter, D. A.: 1998, 'Discovery of Recent Star Formation in the Extreme Outer Regions of Disk Galaxies', Astrophys. J. 506, L19-L22.

Fletcher, A., Beck, R., Berkhuijsen, E. M., Shukurov, A.: 2000, 'The Regular Magnetic Fields in M31 and M33 From Polarization Observations', in E. M. Berkhuijsen, R. Beck, and R. A.M. Walterbos (eds.), The Interstellar Medium in M31 and M33, ShakerVerlag, 2000, pp. 203-206.

Frick, P., Stepanov, R., Shukurov, A., Sokoloff, D.: 2000, 'Structures on the RM Sky', Mon. Not. R. Astron. Soc. in press.

Greaves, J.S., Holland, W.S., Jenness, T., Hawarden, T. G.: 2000, 'Magnetic Field Surrounding the Starburst Nucleus of the Galaxy M82 from Polarized Dust Emission', Nature 404, 732-733.

Han, J. L., and Qiao, G. J.: 1994, 'The Magnetic Field in the Disk of Our Galaxy', Astron. Astrophys. 288, 759-772. 
Han, J. L., and Qiao, G. J.: 1996, 'On Galactic Magnetic Field Derived from RMs of Pulsars', in S. Johnston, M. A. Walker, and M. Bailes (eds.), Pulsars: Problems and Progress, ASP Conf. Ser. Vol. 105, pp. 485-486.

Han, J. L., Manchester, R. N., Berkhuijsen, E. M., Beck, R.: 1997, 'Antisymmetric Rotation Measures in Our Galaxy: Evidence for an A0 Dynamo', Astron. Astrophys. 322, 98102.

Han, J. L., Manchester, R.N., Qiao, G. J.: 1999a, 'Pulsar Rotation Measures and the Magnetic Structure of Our Galaxy', Mon. Not. R. Astron. Soc. 306, 371-380.

Han, J. L., Beck, R., Ehle, M., Haynes, R. F., Wielebinski, R.: 1999b, 'Magnetic Fields in the Spiral Galaxy NGC 2997', Astron. Astrophys. 348, 405-417.

Haslam, C. G. T., Wilson, W. E., Graham, D. A., Hunt, G. C.: 1974, 'A Further 408 MHz Survey of the Northern Sky', Astron. Astrophys. Suppl. 13, 359-394.

Haverkorn, M., Katgert, P., de Bruyn, A. G.: 2000, 'Structure in the Local Galactic ISM on Scales Down to 1 pc, From Multi-Band Radio Polarization Observations', Astron. Astrophys. 356, L13-L16.

Haynes, R.F., Stewart, R.T., Gray, A.D., Reich, W., Reich, P., Mebold, U.: 1992, 'Polarized Arcs Near the Galactic Centre', Astron. Astrophys. 264, 500-512.

Heiles, C.: 1976, 'The Interstellar Magnetic Field', Ann. Rev. Astron. Astrophys. 14, 1-22.

Heiles, C.: 1996, 'A Comprehensive View of the Galactic Magnetic Field, Especially Near the Sun', in W. G. Roberge and D. C. B. Whittet (eds.), Polarimetry of the Interstellar Medium, ASP Conf. Ser. 97, 1996, pp. 457-474.

Hummel, E., van Gorkom, J.H., Kotanyi, C. G.: 1983, 'Anomalous Radio Continuum Features in Edge-on Spiral Galaxies', Astrophys. J. 267, L5-L9.

Indrani, C., and Deshpande, A.A.: 1998, 'The Large Scale Structure of the Galactic Magnetic Field', New Astronomy 4, 33-40.

Junkes, N., Fürst, E., Reich, W.: 1987, 'A Survey of Linear Polarization Along the Galactic Plane', Astron. Astrophys. Suppl. 69, 451-464.

Krause, M.: 1990, 'Multi-frequency Radio Observations of Spiral Galaxies and Their Interpretation', in R. Beck, P.P. Kronberg, and R. Wielebinski (eds.), Galactic and Intergalactic Magnetic Fields, Kluwer Acad. Publ., Dordrecht, 1990, pp. 187-196.

Lang, C. C., Anantharamaiah, K.R., Kassim, N. E., Lazio, T. J. W.: 1999, 'Discovery of a Nonthermal Galactic Center Filament Parallel to the Galactic Plane', Astrophys. J. 521, L41-44.

Lesch, H., and Reich, W.: 1992, 'The Origin of Monoenergetic Electrons in the Arc of the Galactic Center. Particle Acceleration by Magnetic Reconnection', Astron. Astrophys. 264, 493-499.

Minter, A. H., and Spangler, S. R.: 1996, 'Observation of Turbulent Fluctuations in the Interstellar Plasma Density and Magnetic Field on Spatial Scales of 0.01 to 100 parsecs', Astrophys. J. 458, 194-214.

Morris, M.: 1994, 'Magnetic Phenomena', in R. Genzel and A. I. Harris (eds.), The Nuclei of Normal Galaxies, Kluwer Acad. Publ., Dordrecht, 1994, pp. 185-198.

Niklas, S.: 1995, 'Eigenschaften von Spiralgalaxien im hochfrequenten Radiokontinuum', Ph.D. Thesis, University of Bonn.

Niklas, S., and Beck, R.: 1997, 'A New Approach for the Radio-far Infrared Correlation for Non-calorimeter Galaxies', Astron. Astrophys. 320, 54-64.

Ohno, H., and Shibata, S.: 1993, 'The Random Magnetic Field in the Galaxy', Mon. Not. R. Astron. Soc. 262, 953-962.

Phillipps, S., Kearsey, S., Osborne, J. L., Haslam, C. G. T., and Stoffel, H.: 1981, 'Distribution of Galactic Synchrotron Emission', Astron. Astrophys. 98, 286-294.

Plante, R. L., Lo, K.Y., Crutcher, R.M., Killeen, N.E. B.: 1994, 'The Magnetic Field at the Galactic Center: Detection of HI Zeeman Splitting', in R. Genzel and A.I. 
Harris (eds.), The Nuclei of Normal Galaxies, Kluwer Acad. Publ., Dordrecht, 1994, pp. 205-207.

Poezd, A., Shukurov, A., Sokoloff, D.: 1993, 'Global Magnetic Patterns in the Milky Way and the Andromeda Nebula', Mon. Not. R. Astron. Soc. 264, 285-297.

Pohl, M.: 1993, 'On the Predictive Power of the Minimum Energy Condition I. Isotropic Steady-state Configurations', Astron. Astrophys. 270, 91-101.

Pohl, M., Reich, W., Schlickeiser, R.: 1992, 'Synchrotron Modelling of the 400 pc Spur at the Galactic Center', Astron. Astrophys. 262, 441-454.

Rand, R. J., and Kulkarni, S. R.: 1989, 'The Local Galactic Magnetic Field', Astrophys. J. 343, 760-772.

Rand, R. J., and Lyne, A. G.: 1994, 'New Rotation Measures of Distant Pulsars in the Inner Galaxy and Magnetic Field Reversals', Mon. Not. R. Astron. Soc. 268, 497-505.

Reich, W.: 1994, 'High Frequency Radio Observations of Polarized Emission in the Galactic Center', in R. Genzel and A. I. Harris (eds.), The Nuclei of Normal Galaxies, Kluwer Acad. Publ., Dordrecht, 1994, pp. 55-62.

Reich, W., Sofue, Y., Matsuo, H.: 2000, '150 GHz NOBA Observations of the Galactic Center Arc', Publ. Astron. Soc. Japan 52, 355-361.

Rohde, R., Beck, R., Elstner, D.: 1999, 'Magnetic Arms in NGC 6946 Generated by a Turbulent Dynamo', Astron. Astrophys. 350, 423-433.

Schlickeiser, R.: 1984, 'An Explanation of Abrupt Cutoffs in the Optical-infrared Spectra of Non-thermal Sources. A New Pile-up Mechanism for Relativistic Electron Spectra', Astron. Astrophys. 136, 227-236.

Seiradakis, J. H., Lasenby, A. N., Yusef-Zadeh, F., Wielebinski, R., Klein, U.: 1985, 'A New Symmetrical Polarization Structure Near the Galactic Centre', Nature 317, 697-699.

Simard-Nomandin, M., and Kronberg, P. P.: 1980, 'Rotation Measures and the Galactic Magnetic Field', Astrophys. J. 242, 74-94.

Sokoloff, D.D., Bykov, A.A., Shukurov, A., Berkhuijsen, E. M., Beck, R., Poezd, A.D.: 1998, 'Depolarization and Faraday Effects in Galaxies', Mon. Not. R. Astron. Soc. 299, 189-206 and Mon. Not. R. Astron. Soc. 303, 207-208 (Erratum).

Spoelstra, T. A. Th.: 1977, 'The Galactic Magnetic Field', Sov. Phys. Usp. 20, 336-342.

Strong, A. W., Moskalenko, I. V., Reimer, O.: 2000, 'Diffuse Continuum Gamma Rays from the Galaxy', Astrophys. J. 537, 763-784.

Taylor, J. H., and Cordes, J. M.: 1993, 'Pulsar Distances and the Galactic Distribution of Free Electrons', Astrophys. J. 411, 674-684.

Tinbergen, J.: 1996, Astronomical Polarimetry, Cambridge Univ. Press.

Tsuboi, M., Inoue, M., Handa, T., Tabara, H., Kato, T., Sofue, Y., Kaifu, N.: 1986, 'Prominent Polarized Plumes in the Galactic Center Region and Their Magnetic Field', Astron. J. 92, 818-824.

Uchida, K.I., and Güsten, R.: 1995, 'The Large-scale Magnetic Field in the Galactic Center', Astron. Astrophys. 298, 473-481.

Uyanıker, B., Fürst, E., Reich, W., Reich, P., Wielebinski, R.: 1999, 'A 1.4 GHz Radio Continuum and Polarization Survey at Medium Galactic Latitudes', Astron. Astrophys. Suppl. 138, 31-45.

Vallée, J. P.: 1995, 'The Milky Way's Spiral Arms Traced by Magnetic Fields, Dust, Gas, and Stars', Astrophys. J. 454, 119-124.

Vallée, J.P.: 1996, 'Magnetic Field Reversals in the Milky Way — "Cherchez le Champ Magnétique"', Astron. Astrophys. 308, 433-440.

Verschuur, G. L.: 1979, 'Observations of the Galactic Magnetic Field', Fund. Cosmic Phys. 5, 113-191. 
Yusef-Zadeh, F., Roberts, D. A., Goss, W. M., Frail, D. A., Green, A. J.: 1996, 'Detection of $1720 \mathrm{MHz}$ Hydroxyl Masers at the Galactic Center: Evidence for Shock-excited Gas and Milligauss Fields', Astrophys. J. 466, L25-L29.

Address for Offprints: Max-Planck-Institut für Radioastronomie, Auf dem Hügel 69, D53121 Bonn, Germany; rbeck@mpifr-bonn.mpg.de 